\title{
A TiN@Au-NR Plasmonic Structure with Tunable Surface Plasmon Resonance Depending on TiN to Au Thickness Ratio
}

\author{
Ayoub Laghrissi ${ }^{1} \cdot$ Mohammed Es-Souni $^{1}$ (D)
}

Received: 10 June 2020 / Accepted: 4 August 2020 / Published online: 15 August 2020

(C) The Author(s) 2020

\begin{abstract}
TiN nanostructures have been shown to exhibit promising plasmonic properties and are potential candidates for various applications, including energy harvesting. However, these properties also show a strong dependence on the processing conditions which have been reported to affect metallicity of TiN. Herein, we report on layered TiN@Au-nanorods (NRs) nanostructures consisting of $20 \mathrm{~nm}$ TiN thin layer that is magnetron sputtered on Au-NRs of variable length, yielding different TiN/Au thickness ratios $(\mathrm{R})$. While a $20 \mathrm{~nm}$ TiN layer sputtered on the same substrate on which the Au-NRs are grown shows a weak absorption peak in the near IR region, an intense and broad plasmonic peak that lies red from the transverse plasmonic peak of monolithic Au-NRs layer is observed for TiN@Au-NRs. The red-shift is shown to increase with increasing R, attaining $100 \mathrm{~nm}$ for R=1, together with an intense tail in the IR region. These results are interpreted in terms of a strong coupling between TiN and Au that drastically affects the plasmonic behavior of the structure. The results are contrasted with those on Pd@Au-NRs where only a slight blue-shift of few nanometers from the Au peak is observed. Potential applications of the TiN@Au-NRs are mainly in energy harvesting such as water splitting and photocatalysis using electromagnetic radiation in a broad wavelength range, as well as medical applications. Pd@Au-NRs may be used as electrocatalysts with plasmonic enhancement, e.g., for the hydrogen evolution reaction.
\end{abstract}

Keywords Composite 1D-nanostructures · TiN layer on Au-nanorods · Pd layer on Au-nanorods · Plasmonic absorption · Tunable plasmonics · FDTD

\section{Introduction}

The most widely known and applied plasmonic nanostructures are based on the noble metals $\mathrm{Ag}$ and $\mathrm{Au}$ that exhibit localized surface plasmon resonance (LSPR) which is a collective dipolar oscillation of conduction electrons in phase with the incident electromagnetic field. Plasmonic nanostructures find a wide range of applications spanning the fields of biosensing and energy harvesting to photocatalysis [1-7]. In the last few years, however, transition metal nitrides showing metallic conduction, prominent among them is TiN, have seen a flurry of research activities as emerging plasmonic

Electronic supplementary material The online version of this article (https://doi.org/10.1007/s11468-020-01254-z) contains supplementary material, which is available to authorized users.

Mohammed Es-Souni

mohammed.es-souni@fh-kiel.de

1 Institute for Materials \& Surface Technology, University of Applied Sciences, Grenzstrasse 3, D-24149 Kiel, Germany nanomaterials with properties purportedly surpassing those of noble metals [8]. Recently a few applications have been demonstrated using $\mathrm{TiN}$ as a plasmonic nanomaterial for hot carrier injection in the wide visible range of the optical spectrum. For instance, Ishi et al. [9] report on a TiN/ZnO/TiN structure that shows a higher photocatalytic performance under (simulated) sunlight, with photocurrent responses surpassing those of $\mathrm{Au} / \mathrm{ZnO} / \mathrm{Au}$ [9]. TiN is also CMOS (complementary metal-oxide-semiconductor) compatible and has been recently reported for self-powered photodetectors when used as a backside illuminated TiN layer on p-type silicon [10].

There are undeniable advantages of TiN that understandably accrue from its high hardness (for instance, TiN is widely known as a hard coating for high speed steel tools) and corrosion resistance which should yield robust plasmonic devices. However, the optical and also the electronic properties of TiN very much depend on the processing conditions which control the amount of impurities, mostly oxygen, and particle size. For instance, Yick et al. show that surface chemistry and particle size of TiN nanoparticles (NPs), fabricated by the transferred arc plasma technique, affect the plasmonic peak 
position and its shape [11]. In particular, they demonstrate that enhancing the metallic character of the NPs through high applied currents results in a blue-shift of the plasmonic peak. TiN films with the "best" plasmonic behavior were claimed by Sugavaneshwar et al. who used pulsed laser deposition on silicon and polymeric substrates [12]. Their analytically calculated scattering and absorption spectra point to plasmonic properties similar to those of Au-NPs.

Nanostructured $\mathrm{Au}$ is a well-established plasmonic material, while TiN is an emerging one with plasmonic properties that depend on the chemistry achieved (see above). Questions arise as to how the plasmonic properties will change in the case of hybridTiN@Au nanostructures and what are the means to control them. It is the aim of the present work to address these questions. For this purpose, we investigate the plasmonic properties of TiN-NPs@Au-NRs as a function of TiN to Au-NR thickness ratio (R) and show a unique plasmonic absorption behavior that arises from the coupling between TiN-NPs and Au-NRs. Depending on R, the plasmonic absorption red-shifts by up to $100 \mathrm{~nm}$, along with a high background that extends into the NIR region. We contrast these results with those obtained on Pd-NPs coated Au-NR having the same $\mathrm{Au} / \mathrm{Pd}$ ratio, where rather a blue-shift of few nanometers of the absorption peak was observed. For the purpose of this study, we fabricated Au-NRs of different length using electrodeposition in anodized aluminum oxide (AAO) template films on glass substrates. After exposing the Au-NR structure, TiN and Pd films of $20 \mathrm{~nm}$ thickness were sputtered on them via physical vapor deposition.

\section{Materials and Methods}

\section{Synthesis and Characterization}

The following chemicals were used as purchased: oxalic acid dihydrate 99\% (Roth, Karlsruhe, Germany), phosphoric acid 85\% (Roth, Karlsruhe, Germany), sodium hydroxide (Roth, Karlsruhe, Germany). and gold(III) chloride trihydrate (Sigma-Aldrich, Steinheim, Germany). Deionized water was used to prepare aqueous solutions.

Aluminum thin films of approximately $500 \mathrm{~nm}$ were deposited on a borosilicate glass/Ti $(2 \mathrm{~nm}) / \mathrm{Au}(5 \mathrm{~nm})$ heterostructure via electron beam evaporation (PVD75, Lesker, Jefferson Hills, USA, equipped with an electron beam system (Ferrotec GmbH, Unterensingen, Germany). Anodization was conducted potentiostatically in $0.1 \mathrm{M}$ oxalic acid at $70 \mathrm{~V}$ in a two-electrode set-up with a Pt mesh as the counter electrode, using an electrochemical workstation (Keithley 2400 SM, Cleveland, USA). After anodization, barrier layer removal and pore widening were performed in phosphoric acid (5 wt. $\%$ in water) for $50 \mathrm{~min}$ at $30^{\circ} \mathrm{C}[13,14]$. AuNRs were grown into the AAO pores via electrodeposition in
$8 \mathrm{mM} \mathrm{HAuCl}_{4}$ aqueous electrolyte using a three-electrode setup, with the Au/AAO template working electrode, a Pt counter electrode, and an $\mathrm{Ag} / \mathrm{AgCl}$ reference electrode, under potentiostatic conditions $(0.1 \mathrm{~V})$ using an electrochemical workstation (Princeton Potentiostat/Galvanostat Model 263A, Oak Ridge, USA). The Au-NRs length was adjusted via controlling deposition time. After electrodeposition, the 1D nanostructures were exposed by dissolving the AAO film in an aqueous solution of $\mathrm{NaOH}(5 \mathrm{wt} . \%)$ at room temperature. Subsequently, the supported $\mathrm{Au}-\mathrm{NRs}$ of different lengths were introduced together with a glass/Ti $(2 \mathrm{~nm}) / \mathrm{Au}$ substrate (the same substrate used to grow the Au-NRs) into the sputtering chamber. Twenty-nanometer-thick TiN and Pd films were RF sputtered on the Au-NRs samples and the supporting substrate using pure targets (TiN (Kurt Lesker Company, Jefferson Hills, USA) and Pd (ESG Edelmetall $\mathrm{GmbH}$, Rheinstetten, Germany)) under an approximate argon partial pressure of $10^{-3} \mathrm{mbar}$. The sputtering power was set to $120 \mathrm{~W}$ for Pd at room temperature and $220 \mathrm{~W}$ for TiN at $250^{\circ} \mathrm{C}$. The film thickness was monitored by a quartz crystal microbalance. It should be pointed out that the sputtering of $20 \mathrm{~nm}$ TiN (or Pd) was conducted simultaneously, under the same conditions for all the samples under study.

The microstructure and morphology of the samples were characterized with a high-resolution scanning electron microscope (SEM Ultra Plus, ZEISS, Oberkochen, Germany) operating in the secondary (SE) and energy selective backscattered (ESB) electron modes. The SEM is also equipped with an energy dispersive X-ray spectroscopy (EDS) package (INCAx-act, Oxford Instruments, High Wycombe, UK). The structure was characterized by X-ray diffraction (XRD, X'Pert Pro diffractometer PANalytical, Eindhoven, Netherland) in grazing incidence diffraction mode with constant $\theta=1^{\circ}$ using monochromatic $\mathrm{CuK} \alpha$ radiation with $\lambda=1.5418 \AA$. The device has a full width to half maximum resolution of $0.03^{\circ}$. The absorption spectra were recorded using a UV-Vis-NIR system in transmission mode (Lambda 950 UV-Vis-NIR Spectrometer, Perkin Elmer, Llantrisant, UK). The substrate (glass/Ti $(2 \mathrm{~nm}) / \mathrm{Au}(5 \mathrm{~nm}))$ was used as reference. X-ray photoelectron spectra were obtained on a TiN film using Omicron full lab system (Scienta Omicron GmbH, Taunusstein, Germany) and $\mathrm{Al} \mathrm{K}_{\mathrm{a}}$ radiation. The films were first cleaned using argon ions sputtering before analysis.

\section{Finite-Difference-Time-Domain (FDTD) Calculations}

The FDTD method implemented in MEEP code was used to calculate the absorption and the field enhancement of Au-NR, Pd@Au-NR, and TiN@Au-NR. The cylindrical symmetry was used to model the structures, and the dielectric functions were described by Lorentz-Drude model. The dielectric function parameters of the different materials were obtained from selected sources: those of TiN were fitted by Patsalas et al. 
$[15,16]$; those of Au were reported by Hao et al. [17] and those of Pd by Rakić et al. [18]. The Au-NR diameter was set to $80 \mathrm{~nm}$, and its length was varied between 20 and $200 \mathrm{~nm}$. The (Pd-) TiN layer was modeled as a disk of $80 \mathrm{~nm}$ dimeter and $20 \mathrm{~nm}$ thickness (see Fig. S1 (supplementary material) for a schematic representation of the model).

\section{Results and Discussion}

\section{Structure and Optical Properties}

Table 1 lists the different TiN/Au and $\mathrm{Pd} / \mathrm{Au}$ thickness ratios investigated. The results are compared with those of a $20 \mathrm{~nm}$ TiN and Pd thin films on the same substrate heterostructure, deposited under the same conditions.

Figure 1a illustrates the nanoparticulate structure of the TiN film with an NP size distribution between 5 and $20 \mathrm{~nm}$. In the layered structure, the two phases are well distinguished through their atomic number contrast in the high-resolution backscatter electron micrograph (Fig. 1b). An exemplary cross-sectional micrograph with a TiN/Au-NRs thickness ratio of 1 is displayed in Fig. 1c. Figure 1d also shows an exemplary high-resolution micrograph of Pd@Au-NRs with a thickness ratio of 0.1. The XRD patterns of the TiN and Pd layers are displayed in Fig. 2. Figure 2a shows a broad TiN 111 peak, with a shift of $0.25^{\circ}$ from the standard card $(00-038$ $\left.14202 \theta_{111}=36.663^{\circ}\right)$. This shift suggests the presence of oxygen defects in the TiN layer (for instance, the standard card no. 01-087-0630 shows a shift of $0.2^{\circ}(2 \theta)$ for the 111peak corresponding to $\left.(\mathrm{TiN})_{0.88} 2 \theta_{111}=36.862^{\circ}\right)$. Using the Scherrer formula, the mean crystallite size amounts to $15 \mathrm{~nm}$ for TiN and $16 \mathrm{~nm}$ for Pd, assuming a shape factor, $\mathrm{K}=1$ (see also the EDS analysis Fig. S2 (supplementary material)).

The absorption spectra of the Au-NRs before and after coating with the TiN and Pd layers are illustrated in Fig. 3. The transverse plasmonic peak of the Au-NRs lies at its usual value of $518 \mathrm{~nm}$ for the $200 \mathrm{~nm}$ long NR (Fig. 3a). Decreasing the Au-NR length (decreasing the aspect ratio), e.g., to $20 \mathrm{~nm}$, leads to a slight red-shift of approximately $40 \mathrm{~nm}$ of the maximum together with a noticeable broadening of the peak and diminishing intensity. It should be mentioned that the longitudinal plasmonic peak was not observed due to the

Table 1 The investigated TiN/Au thickness ratios (R)

\begin{tabular}{ccc}
\hline $\mathrm{R}=\mathrm{TiN} / \mathrm{Au}(\mathrm{Pd} / \mathrm{Au})$ & Au-NRs length $(\mathrm{nm})$ & TiN/Pd thickness $(\mathrm{nm})$ \\
\hline 0.10 & 200 & 20 \\
0.20 & 100 & 20 \\
0.40 & 50 & 20 \\
1.00 & 20 & 20 \\
\hline
\end{tabular}

experimental set-up which allows measurement only parallel to the NR long axis. The $20 \mathrm{~nm}$ TiN layer shows a weak absorption peak at approximately $868 \mathrm{~nm}$ which is somewhat higher than the experimental values reported by Yick et al. for TiN particles processed under different conditions and suspended in water [11]. Both our value and those of Yick et al. [11] are, however, largely beyond the calculated values of Sugavaneshwar et al. for their "best" TiN films [12]. The Pd-NP layer shows only an intense background in the whole wave length range investigated (Fig. S3, supplementary material). At R=0.1, TiN@Au-NR shows a double peak that upon comparison with that of pure Au-NR of the same length is a clear indication of a strong coupling between the Au-NRs and the TiN layer. Deconvolution of the double peak yields a slightly red-shifted Au peak at $524 \mathrm{~nm}$; if we attribute the second peak to TiN, a substantial blue-shift of its plasmonic peak, which now lies at $563 \mathrm{~nm}$, can be suggested. Further, we notice a damping of the plasmonic absorption of the Au-NRs together with a supposedly strong increase in the absorption peak intensity of TiN. For $\mathrm{R}=0.2$, a broad absorption peak is obtained that appears to be composed of two smeared maxima (see inset of Fig. 3d), but the peak positions are rather similar. For $\mathrm{R}>0.2$, the broad absorption peaks observed show a substantial red-shift; e.g., for $\mathrm{R}=1$, the peak position lies at $640 \mathrm{~nm}$, and an intense tail that extends into the near IR region can be noted (compare the dashed-line curves to the continuous line ones). This absorption in the near-infrared region makes these nanomaterials particularly interesting for biomedical devices requiring wavelengths in the range of 1000 $1200 \mathrm{~nm}$ where the human skin is transparent $[19,20]$. The behavior above completely differs from that of the Pd@AuNR nanostructures of similar R, where barely any difference in the absorption wavelength with increasing $\mathrm{R}$ can be noticed, lest for an overall damping of the absorption peak intensity. The Pd@Au-NR results are in agreement with those published by Xiang et al. on Au-NR -core-Pd- shell structures [21], where Pd has been shown to blue-shift the plasmonic absorption peaks of Au-NRs and dampen their intensity, depending on Pd thickness.

The results above, both in terms of peak shift and intensity, unambiguously suggest that there is a strong coupling between the plasmonic absorptions of $\mathrm{Au}$ and TiN, essentially resulting in a behavior completely different from that of monolithic Au-NRs. While a plethora of work has been published on hybrid nanostructures, including $\mathrm{Ag} / \mathrm{Au}$ particles and $\mathrm{Au}$ nanorods as well as $\mathrm{Au}(\mathrm{Ag}) /$ dielectrics in solution (e.g., see the review article by Cortie et al. [22]), nothing has been reported so far on the coupling between noble metal nanostructures and transition metal nitrides. We might treat the present experimental findings assuming, first, the existence of two plasmonic peaks of TiN@Au-NR. Deconvoluting the double/broad absorption peaks, we may then obtain one peak corresponding to that of Au-NR that is 
Fig. 1 SEM micrographs: (a) top view of the TiN thin film. It shows the NP size distribution between 5 and $20 \mathrm{~nm}$; (b) crosssectional, high-resolution backscatter electron micrograph of TiN@Au for $\mathrm{R}=0.2$; the darkcontrast caps on the Au-NRs are the TiN phase (atomic number contrast); (c) cross section of TiN@Au-NRs showing TiN layer thickness of $20 \mathrm{~nm}(\mathrm{R}=1)$; and (d) high-resolution micrograph of Pd@Au-NRs for $\mathrm{R}=0.2$ showing the distribution of Pd nanoparticles on the Au-NRs
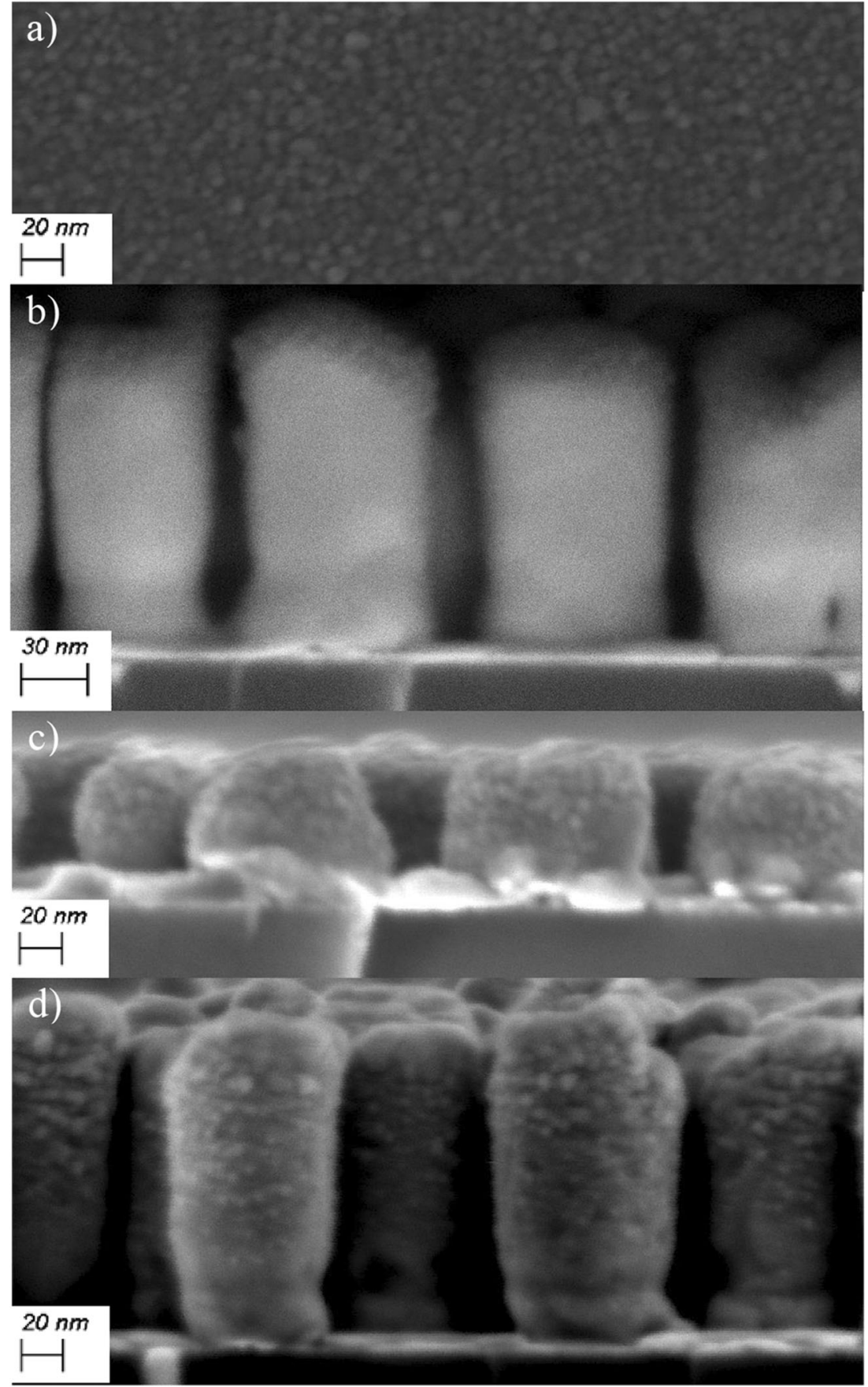

slightly red-shifted, most probably because of the difference in the dielectric permittivity of both materials; the second peak might be attributed to TiN with rather a large blue-shift which might be rationalized in terms of increased metallicity of the TiN layer owing to carrier transfer from $\mathrm{Au}$. Indeed, depending on its chemical composition, TiN is reported with a carrier concentration in the range of $10^{22} / \mathrm{cm}^{3}$. According to Sugavaneshwar et al. [12], increasing metallicity of TiN boosts carrier concentration to approximately $8 \times 10^{22} / \mathrm{cm}^{3}$, and this would lead to plasmonic absorption at wavelengths in the $600 \mathrm{~nm}$ range. Metallic TiN has reportedly a higher
Fermi level and lower work function than $\mathrm{Au}[23,24]$. This should result in electron transfer from $\mathrm{TiN}$ to $\mathrm{Au}$, as depicted in Fig. 4a, that is compensated by hole formation in TiN. However, the material presently dealt with has most probably oxygen defects, as corroborated by its plasmonic peak that lies red-shifted by about $250 \mathrm{~nm}$ from that of a metallic TiN. Further support of the presence of oxygen defects is provided by the XPS spectra of the TiN layer surface, which show oxygen defects (Fig. S4, (supplementary material). Consequently, the presence of oxygen defects could change the material's behavior to a more semi-conducting one, and 
Fig. 2 XRD patterns in grazing incidence mode of the sputtered layers of (a) TiN and (b) Pd on a glass/Ti/Au substrate
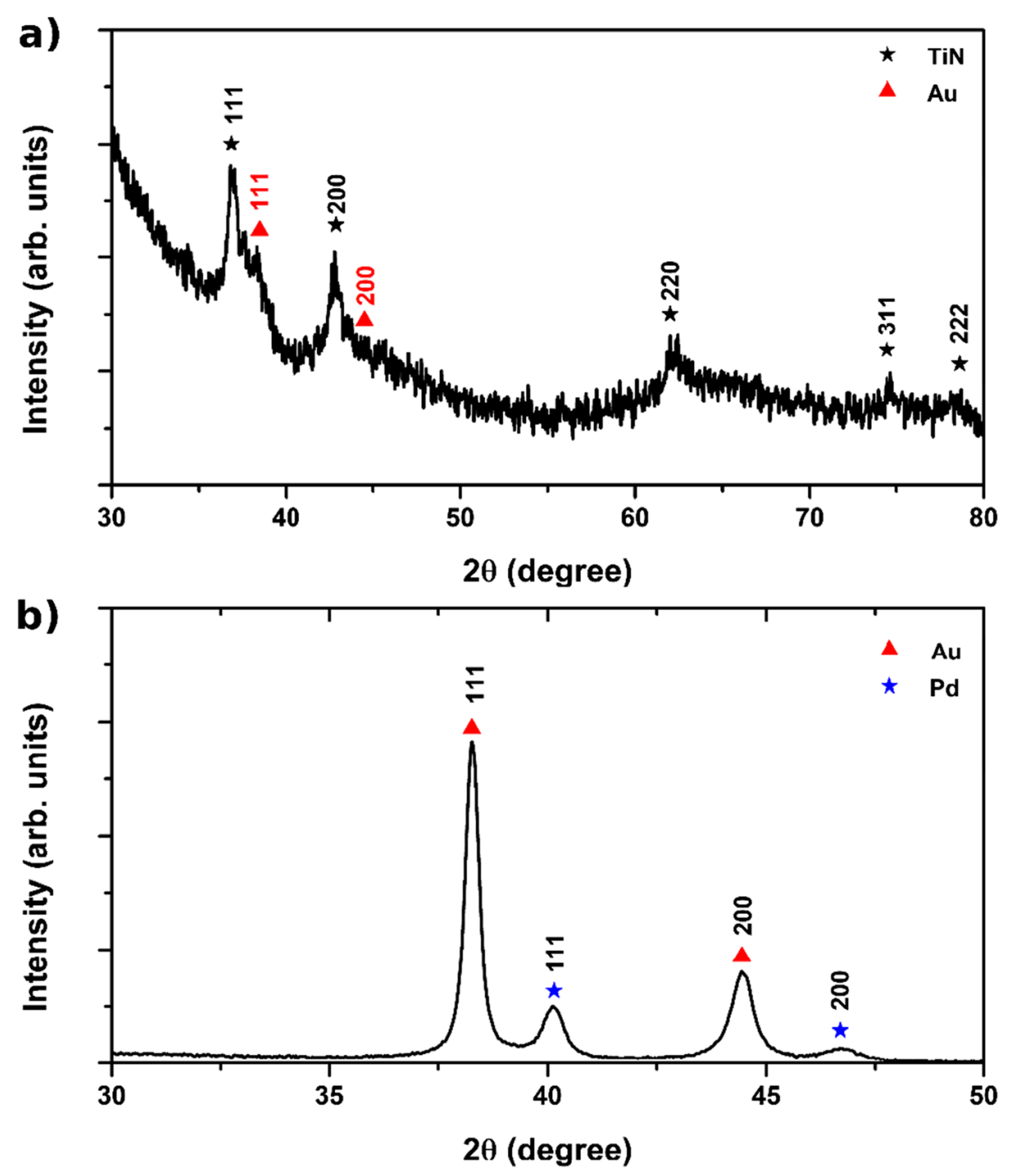

the band diagram in Fig. 4a no more holds. For instance, Lui et al. show that NTiO behaves as a semi-conductor with a band gap that depends on the amount of oxygen defects [25]. Upon excitation, hot electron injection from $\mathrm{Au}$ into the conduction band of the $\operatorname{Ti}(\mathrm{N}, \mathrm{O}) \mathrm{x}$ structure is expected to increase its metallic character (Fig. 4b) that should explain the strong blue-shift of the plasmonic peak of TiN. When $\mathrm{R}$ is increased (this is done by maintaining the TiN thickness at $20 \mathrm{~nm}$ while varying the of the Au-NR length downwards to $20 \mathrm{~nm}$ ), meaning that the nanostructure tends towards Aucore-TiN-shell, the blue-shift of TiN is still substantial, but the peak intensity decreases, while the near-infrared absorption increases over a wide wavelength range. However, it is important to note that, overall, the absorption intensity of all the structures with $\mathrm{R} \geq 0.2$ is higher than that of monolithic Au-NRs (compare Fig. 3a and d) which further confirms the strong coupling between Au and TiN.

In another reasoning, we could attribute the plasmonic absorption behavior of the coupled TiN/Au-NRs structures to the difference in permittivity of both materials that red-shifts the Au plasmonic peak and this to higher wave lengths, depending on $\mathrm{TiN} / \mathrm{Au}-\mathrm{NR}$-thickness ratio. In this case, we assume that the plasmonic absorption of $\mathrm{TiN}$ is overridden by the high background. However, because the TiN layer thickness is maintained constant and the interface between the Au-nanostructures and TiN should not change with decreasing Au-NR length and taking into account the plasmonic absorption behavior of pristine Au-NRs, the strong red-shift increase with increasing $\mathrm{R}$ (approximately $100 \mathrm{~nm}$ for $\mathrm{R}=1$ ) and the higher absorbance observed for the structures with $\mathrm{R}$ $\geq 0.2$ remain to be explained.

Either way, the experimental results above impressively demonstrate the tunability of the plasmonic absorption of TiN@Au-NR structure in a rather large wave length range. The structure is endowed with the additional advantages of chemical and mechanical stability, thanks to the TiN layer, as mentioned in the introduction above.

\section{FDTD Calculations}

The experimental results depicted above were compared with FDTD calculations using the layered model (Fig. S1, supplementary material). We opted for the dielectric function parameters fitted by Patsalas et al. for TiN because they yield a 

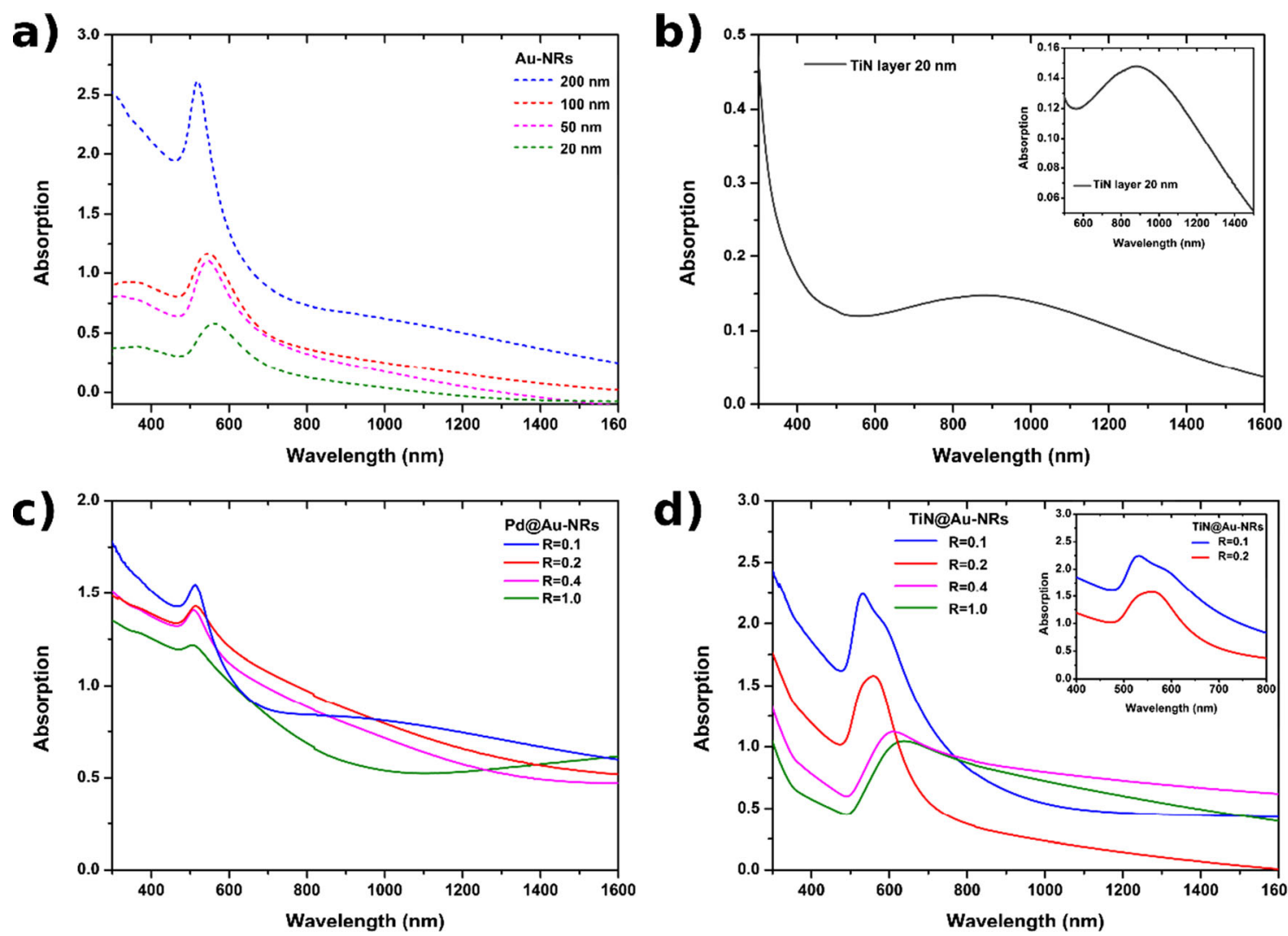

d)

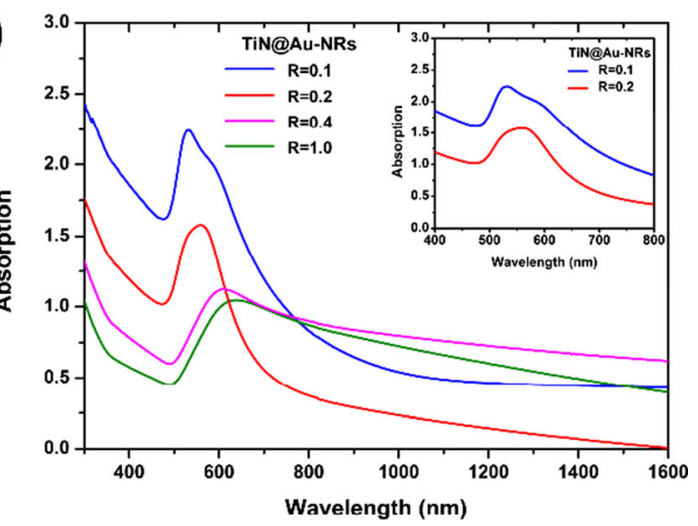

Fig. 3 Measured absorption spectra of (a) Au-NRs before sputtering; (b) $20 \mathrm{~nm}$ thick TiN layer showing a weak peak with a maximum at $868 \mathrm{~nm}$; (c) layered Pd@Au-NR structures of different Pd/Au-NR thickness ratios, R, with a marginal blue-shift of the Au peak; (d) layered TiN@Au-NR structures of different TiN/Au-NR thickness ratios, R. Notice the double peaks for $\mathrm{R}=0.1$ and $\mathrm{R}=0.2$, highlighted in the inset, with a red-shift of

the Au peak and presumably a substantial blue-shift of the TiN peak. For $\mathrm{R} \geq 0.4$, broad absorption peaks can be noticed with tails in the nearinfrared region. At $\mathrm{R}=0.4$, the absorption maximum is at $610 \mathrm{~nm}$, and at $\mathrm{R}=1$, it is at $640 \mathrm{~nm}$. Notice also that, overall, the absorption peak intensity of TiN@Au-NRs is higher than that of Pd@Au-NRs

behavior rather similar to that of $\mathrm{Au}[15,16]$ and, in this respect, reflect a TiN material with high metallicity. This should allow us to study the behavior of the coupled structure. Light absorption of a single pristine Au-NR was calculated, assuming cylindrical symmetry of the NR. The diameter was kept constant at $80 \mathrm{~nm}$, and the nanorod length was changed. This cylindrical symmetry exhibits two plasmon modes: the longitudinal mode where the oscillations of the electrons are along the cylinder axis and a transverse mode for transverse electrons oscillations. Figure S5 (supplementary material) shows the calculated absorption of differently long Au-NR. For the $200 \mathrm{~nm} \mathrm{NR}$, double peaks are observed representing the transversal $(509 \mathrm{~nm})$ and longitudinal $(780 \mathrm{~nm})$ modes. For the $100 \mathrm{~nm}$ NR, the splitting of the plasmonic peaks is not observed due to the low aspect ratio of 1.25; hence, the transversal and longitudinal modes lie in close wavelength range. The calculated absorption peaks are in broad agreement with to the experimental results and with the literature [15]. The absorption of a pure TiN thin disk of $20 \mathrm{~nm}$ thickness and $80 \mathrm{~nm}$ diameter was calculated (Fig. S6, supplementary material); it shows a broad and weak plasmonic peak in agreement with a previous report [16]. The absorption of a Pd thin

disk of $20 \mathrm{~nm}$ thickness and $80 \mathrm{~nm}$ diameter was also calculated. The results depicted in Fig. S7 (supplementary material) show a high background over the visible range with an absorption peak in the UV that is similar to the measured $\mathrm{Pd}$ absorption in the literature [26].

Concerning the layered $\mathrm{Au}-\mathrm{NR} / \mathrm{TiN}(\mathrm{Pd})$ structures, it appeared that the FDTD calculations did not render the behavior experimentally observed. In particular, the coupling between TiN and Au-NRs seemed to be underestimated. The reasons are to be found in the simple layered nanorod model together with the dielectric functions selected from the literature. For instance, comparing the dielectric functions of $\mathrm{Au}$ [17] and $\operatorname{TiN}[15,16]$ reveals the reason behind the nonsignificant difference between the calculated absorptions of Au-NR and layered structures. The real and imaginary parts of the dielectric functions of TiN and Au behave in a similar way with the only difference residing in a higher imaginary part $\left(\varepsilon_{2}\right)$ and a lower quality factor for TiN (broader LSPR peak).

Because the model used is based on the propagation of the electromagnetic wave from one material to the other, with their respective dielectric functions, and does not take into account charge transfer at the interface, the 
Fig. 4 Suggested band diagrams of (a) $\mathrm{Au} /($ metallic) TiN junction where electron transfer from TiN to $\mathrm{Au}$ takes place leaving empty states in TiN (see main text for more details) and (b) $\mathrm{Au} / \mathrm{TiN}$ where oxygen defects change the TiN behavior from metallic to more semi-conducting; the electron transfer in this case takes place from $\mathrm{Au}$ to $\mathrm{TiN}$, changing electron carrier density of $\operatorname{TiN}\left(\mathrm{O}_{\mathrm{x}}\right)$

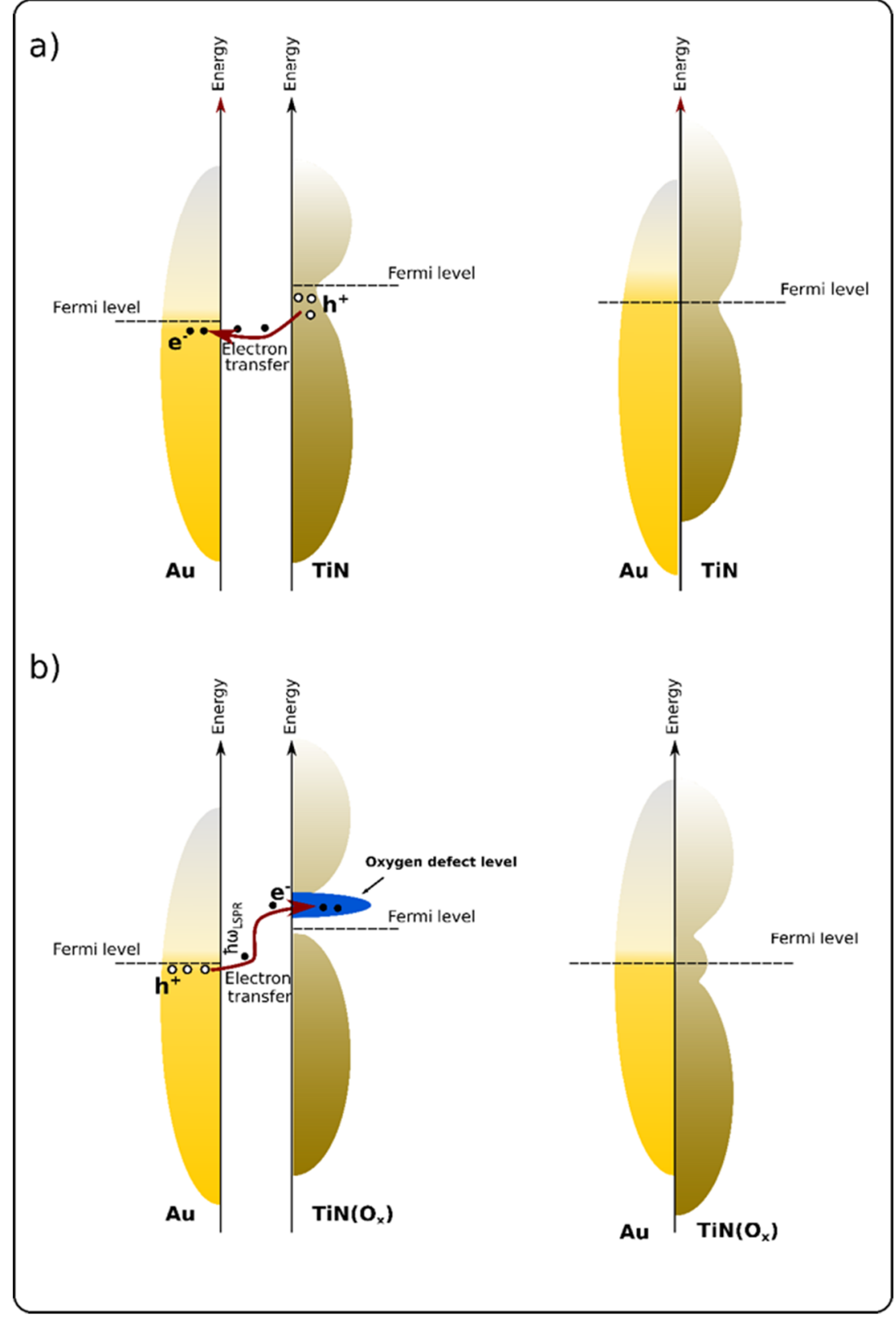

failed calculation results can be explained by the close dielectric functions used for TiN and $\mathrm{Au}$. Further to the limitations of our model, possible coupling between the TiN nanoparticles in the film, e.g., as nanodimers, which can change the optical behavior of the layered nanostructure, is neglected in the calculations.

In order to circumvent the drawback of the model, the dielectric function of the TiN layer was changed to that of a mixed dielectric function of an Au:TiN alloy (1:1) to account for TiN metallicity [27]. The results obtained for TiN@Au for $\mathrm{R}=0.2$ and 1 are displayed in Fig. 5; they show a good agreement with the experimental results shown in Fig. 3 and would support our claim of a strong coupling between $\mathrm{TiN}$ and $\mathrm{Au}$ nanostructures.

\section{Conclusions}

In the present paper, TiN@Au-NRs and Pd@Au-NRs are processed using a combination of electrodeposition of $\mathrm{Au}$ NR in porous anodic aluminum template films on glass substrates and physical vapor deposition of $20 \mathrm{~nm}$ thick TiN and Pd layers. The TiN (Pd) to Au-NRs thickness ratios (R) are tailored by maintaining the TiN (Pd) film thickness of $20 \mathrm{~nm}$ and varying the $\mathrm{Au}-\mathrm{NRs}$ length. A monolithic, nanostructured TiN film sputtered on the same substrate used to grow AuNRs shows a broad and weak plasmonic peak in the NIR region,while TiN@Au-NRs is characterized by an intense plasmonic peak that lies red from the plasmonic peak of $\mathrm{Au}-$ NRs. With increasing R, the peak substantially red-shifts, e.g., 


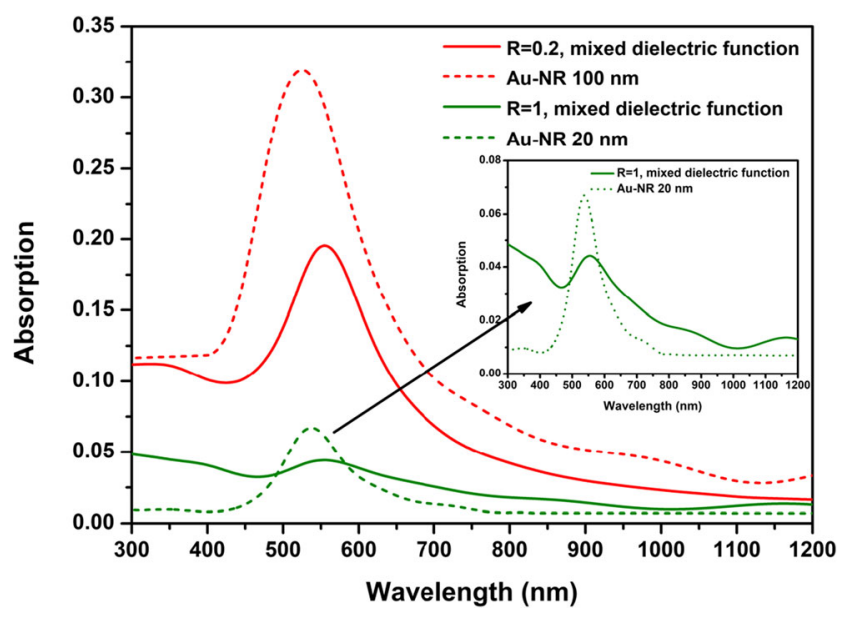

Fig. 5 Calculated absorption of a single Au-NR and layered TiN@AuNR structures with $R=0.2$ and $R=1$, using a mixed dielectric function of a layer consisting of an TiN:Au 1:1 alloy [27]

by $100 \mathrm{~nm}$ for $\mathrm{R}=1$, together with the appearance of a tail in the IR region. This behavior contrasts with that of Pd@AuNRs which shows only a marginal blue-shift of the Au peak. We surmise that the particular plasmonic properties of TiN@Au-NRs are the result of a strong coupling between TiN and Au-NRs, probably involving carrier transfer from $\mathrm{Au}$ to TiN. FDTD calculations using a simple model and dielectric functions from the literature failed to accurately predict the experimental results. However, assuming for TiN, a mixed dielectric function reported for a TiN:Au alloy seems to improve the agreement between experiment and calculations. Applications of the TiN@Au-NRs structures may encompass mechanically robust plasmonic devices with tunable plasmonic absorption, light harvesting, and photocatalytic nanostructures. Pd@Au-NRs may be particularly useful for photoelectrocatalytic applications, e.g., for the electrocatalytic oxidation of formic acid and for the hydrogen evolution reaction using light enhancement.

Acknowledgments The authors thank Dr. Thomas Strunskuss, Technical Faculty, University of Kiel, Germany, for performing the XPS analysis.

Funding Information Open Access funding provided by Projekt DEAL.

\section{Compliance with Ethical Standards}

Conflict of Interest The authors declare that they have no conflict of interest.

Open Access This article is licensed under a Creative Commons Attribution 4.0 International License, which permits use, sharing, adaptation, distribution and reproduction in any medium or format, as long as you give appropriate credit to the original author(s) and the source, provide a link to the Creative Commons licence, and indicate if changes were made. The images or other third party material in this article are included in the article's Creative Commons licence, unless indicated otherwise in a credit line to the material. If material is not included in the article's Creative Commons licence and your intended use is not permitted by statutory regulation or exceeds the permitted use, you will need to obtain permission directly from the copyright holder. To view a copy of this licence, visit http://creativecommons.org/licenses/by/4.0/.

\section{References}

1. Caucheteur C, Guo T, Albert J (2015) Review of plasmonic fiber optic biochemical sensors: improving the limit of detection. Anal Bioanal Chem 407:3883-3897. https://doi.org/10.1007/s00216014-8411-6

2. Špacková B, Wrobel P, Bocková M, Homola J (2016) Optical biosensors based on plasmonic nanostructures: a review. Proc IEEE 104:2380-2408. https://doi.org/10.1109/JPROC.2016. 2624340

3. Choi I, Choi Y (2012) Plasmonic nanosensors: review and prospect. IEEE J Sel Topics Quantum Electron 18:1110-1121. https:// doi.org/10.1109/JSTQE.2011.2163386

4. Ma XC, Dai Y, Yu L, Huang BB (2016) Energy transfer in plasmonic photocatalytic composites. Light: Sci Appl 5:e16017. https:// doi.org/10.1038/lsa.2016.17

5. Hou W, Cronin SB (2013) A review of surface plasmon resonanceenhanced photocatalysis. Adv Funct Mater 23:1612-1619. https:// doi.org/10.1002/adfm.201202148

6. Zhang X, Chen YL, Liu RS, Tsai DP (2013) Plasmonic photocatalysis. Rep Prog Phys 76:046401. https://doi.org/10. 1088/0034-4885/76/4/046401

7. Mandal P, Sharma S (2016) Progress in plasmonic solar cell efficiency improvement: a status review. Renew Sust Energy Rev 65: 537-552. https://doi.org/10.1016/j.rser.2016.07.031

8. Ishii S, Shinde SL, Nagao T (2019) Nonmetallic materials for plasmonic hot carrier excitation. Adv Opt Mater 7:1800603. https://doi. org/10.1002/adom.201800603

9. Ishii S, Shinde SL, Jevasuwan W, Fukata N, Nagao T (2016) Hot electron excitation from titanium nitride using visible light. ACS Photonics 3:1552-1557. https://doi.org/10.1021/acsphotonics. $6 \mathrm{~b} 00360$

10. Güsken NA, Lauri A, Li Y, Matsui T, Doiron B, Bower R, Regoutz A, Mihai A, Petrov PK, Oulton RF, Cohen LF, Maier SA (2019) $\mathrm{TiO}_{2-\mathrm{x}}$-enhanced IR hot carrier based photodetection in metal thin film-Si junctions. ACS Photonics 6:953-960. https://doi.org/10. 1021/acsphotonics.8b01639

11. Yick S, Murdock AT, Martin PJ, Kennedy DF, Maschmeyer T, Bendavid A (2018) Tuning the plasmonic response of TiN nanoparticles synthesised by the transferred arc plasma technique. Nanoscale 10:7566-7574. https://doi.org/10.1039/C7NR09309H

12. Sugavaneshwar RP, Ishii S, Dao TD, Ohi A, Nabatame T, Nagao T (2018) Fabrication of highly metallic TiN films by pulsed laser deposition method for plasmonic applications. ACS Photonics 5: 814-819. https://doi.org/10.1021/acsphotonics.7b00942

13. Es-Souni M, Habouti S (2014) Ordered nanomaterial thin films via supported anodized alumina templates. Front Mater 1:19. https:// doi.org/10.3389/fmats.2014.00019

14. Berger N, Es-Souni M (2016) Understanding and shaping the morphology of the barrier layer of supported porous anodized alumina on gold underlayers. Langmuir 32:6985-6990. https://doi.org/10. 1021/acs.langmuir.6b01732

15. Patsalas P, Kalfagiannis N, Kassavetis S (2015) Optical properties and plasmonic performance of titanium nitride. Materials 8:3128 3154. https://doi.org/10.3390/ma8063128

16. Patsalas P, Logothetidis S (2001) Optical, electronic, and transport properties of nanocrystalline titanium nitride thin films. J Appl Phys 90:4725-4734. https://doi.org/10.1063/1.1403677 
17. Hao F, Nordlander P (2007) Efficient dielectric function for FDTD simulation of the optical properties of silver and gold nanoparticles. Chem Phys Lett 446:115-118. https://doi.org/10.1016/j.cplett. 2007.08.027

18. Rakić AD, Djurišić AB, Elazar JM, Majewski ML (1998) Optical properties of metallic films for vertical-cavity optoelectronic devices. Appl Opt 37:5271-5283. https://doi.org/10.1364/AO.37. 005271

19. Tao Z, Hong G, Shinji C, Chen C, Diao S, Antaris AL, Zhang B, Zou Y, Dai H (2013) Biological imaging using nanoparticles of small organic molecules with fluorescence emission at wavelengths longer than $1000 \mathrm{~nm}$. Angew Chem Int Ed 52:13002-13006. https://doi.org/10.1002/anie.201307346

20. Troy TL, Thennadil SN (2001) Optical properties of human skin in the near infrared wavelength range of 1000 to $2200 \mathrm{~nm}$. J Biomed Opt 6:167-176. https://doi.org/10.1117/1.1344191

21. Xiang Y, Wu X, Liu D, Jiang X, Chu W, Li Z, Ma Y, Zhou W, Xie $S$ (2006) Formation of rectangularly shaped $\mathrm{Pd} /$ au bimetallic nanorods: evidence for competing growth of the Pd shell between the $\{110\}$ and $\{100\}$ side facets of au Nanorods. Nano Lett 6:2290 2294. https://doi.org/10.1021/n1061722c

22. Cortie MB, McDonagh M (2011) Synthesis and optical properties of hybrid and alloy plasmonic nanoparticles. Chem Rev 111:37133735. https://doi.org/10.1021/cr1002529

23. Gosciniak J, Atar FB, Corbett B, Rasras M (2019) Plasmonic Schottky photodetector with metal stripe embedded into semiconductor and with a CMOS-compatible titanium nitride. Sci Rep 9:6048. https://doi.org/10.1038/s41598-019-42663-3

24. Wang X, Jian J, Diaz-Amaya S, Kumah CE, Lu P, Huang J, Lim DG, Pol VG, Youngblood JP, Boltasseva A, Stanciu LA, O'Carroll DM, Zhang X, Wang H (2019) Hybrid plasmonic Au-TiN vertically aligned nanocomposites: a nanoscale platform towards tunable optical sensing. Nanoscale Adv 1:1045-1054. https://doi.org/ $10.1039 / \mathrm{c} 8 \mathrm{na} 00306 \mathrm{~h}$

25. Liu B, Wen L, Zhao X (2008) The structure and photocatalytic studies of $\mathrm{N}$-doped $\mathrm{TiO}_{2}$ films prepared by radio frequency reactive magnetron sputtering. Sol Energy Mater Sol C 92:1-10. https://doi. org/10.1016/j.solmat.2007.07.009

26. Larios-Rodríguez EA, Castillón-Barraza FF, Borbón-González DJ, Herrera-Urbina R, Posada-Amarillas A (2013) Green-chemical synthesis of monodisperse $\mathrm{Au}, \mathrm{Pd}$ and bimetallic (Core-Shell) Au-Pd, Pd-Au nanoparticles. Adv Sci Eng Med 5:665-672. https://doi.org/ 10.1166/asem.2013.1354

27. Ma H, Liu X, Gao C, Yin Y (2020) The calculated dielectric function and optical properties of bimetallic alloy nanoparticles. J Phys Chem C 124:2721-2727. https://doi.org/10.1021/acs.jpcc.9b11154

Publisher's Note Springer Nature remains neutral with regard to jurisdictional claims in published maps and institutional affiliations. 